Photoexcited-induced sensitivity of InGaAs surface QDs to environment

This content has been downloaded from IOPscience. Please scroll down to see the full text. 2014 Nanotechnology 25445501

(http://iopscience.iop.org/0957-4484/25/44/445501)

View the table of contents for this issue, or go to the journal homepage for more

Download details:

IP Address: 138.100.16.180

This content was downloaded on 22/10/2014 at 08:41

Please note that terms and conditions apply. 


\title{
Photoexcited-induced sensitivity of InGaAs surface QDs to environment
}

\author{
M J Milla, J M Ulloa and A Guzmán \\ Instituto de Sistemas Optoelectrónicos y Microtecnología (ISOM), Dpto. Ingeniería Electrónica, \\ Universidad Politécnica de Madrid, Ciudad Universitaria s/n, 28040 Madrid, Spain \\ E-mail: mjmilla@die.upm.es
}

Received 23 June 2014, revised 1 September 2014

Accepted for publication 11 September 2014

Published 17 October 2014

\begin{abstract}
A detailed analysis of the impact of illumination on the electrical response of $\operatorname{In}_{0.5} \mathrm{Ga}_{0.5} \mathrm{As}$ surface nanostructures is carried out as a function of different relative humidity conditions. The importance of the surface-to-volume ratio for sensing applications is once more highlighted. From dark-to-photo conditions, the sheet resistance (SR) of a three-dimensional $\operatorname{In}_{0.5} \mathrm{Ga}_{0.5} \mathrm{As}$ nanostructure decays two orders of magnitude compared with that of a two-dimensional nanostructure. The electrical response is found to be vulnerable to the energy of the incident light and the external conditions. Illuminating with high energy light translates into an SR reduction of one order of magnitude under humid atmospheres, whereas it remains nearly unchanged under dry environments. Conversely, lighting with energy below the bulk energy bandgap, shows a negligible effect on the electrical properties regardless the local moisture. Both illumination and humidity are therefore needed for sensing. Photoexcited carriers can only contribute to conductivity if surface states are inactive due to water physisorption. The strong dependence of the electrical response on the environment makes these nanostructures very suitable for the development of highly sensitive and efficient sensing devices.
\end{abstract}

Keywords: quantum dots, surface states, photoexcitation, humidity sensors, adsorption

(Some figures may appear in colour only in the online journal)

\section{Introduction}

Solid-state semiconductors have been extensively investigated due to their exceptional optical and electronic properties, in addition to their eminent potential for optoelectronic applications. In recent decades, it has been also shown their great suitability for the development of environmental sensing devices, since the contact with the atmosphere yields significant changes in the physical properties of their surface $[1,2]$. Materials such as porous $\mathrm{Si}[3], \mathrm{ZnO}$ nanostructures [4], colloidal II-VI quantum dots (QDs), graphene [5], carbon QDs [6] and III-V semiconductors [7-9] have been proposed as candidates for the development of environmental sensing devices. In particular, surface compound nanostructures based on the high mature arsenide technology (III-As) have recently reported a high sensitivity to external conditions [10-13]. The strong correlation between external conditions and the optical properties of the InGaAs surface QD (SQD) has been addressed to rely on the coupling between surface and confined states [14-17]. PL intensity quenches under vacuum and decreases in dry environments, whereas it is maintained under water-vapor-containing atmospheres [11]. Features of molecules in the surroundings, such as molecular weight, polar character and size among others, have been suggested to be responsible for similar effects $[18,19]$.

Conversely, little work is found in the current literature regarding the electrical response of InGaAs SQDs. Recent studies have shown the strong impact of the relative humidity (RH) in the atmosphere on the SR [13], i.e. the conductivity increases in high $\mathrm{RH}$ environments. Nonetheless, little is known about the effect of illumination on the electrical response of SQDs. Determining the role of light could help researchers better understand the conduction mechanism, thus improving the quality of the sensor and allowing the development of more sensitive and efficient sensing devices.

In this work, we evaluate the impact of the illumination on the electrical response of InGaAs surface nanostructures: 
quantum wells (SQW) and SQDs. We conduct a narrow analysis of the I-V characteristics as a function of the relative humidity $(\mathrm{RH})$ in the atmosphere under the incident light of different energy: infrared light emitting at $1.3 \mu \mathrm{m}(\sim 0.95 \mathrm{eV})$, in the range of the SQD band-to-band energy and, therefore, below GaAs bulk $\left(E_{g, G a A s}\right)$, and visible light above the $E_{g}$, GaAs, respectively. We show that the energy of the incident light plays a significant role in the electrical response. Infrared light $\left(\mathrm{h} \nu<\mathrm{E}_{\mathrm{g}, \mathrm{GaAs}}\right)$ does not affect the $\mathrm{I}-\mathrm{V}$ response in spite of the external humidity conditions; the few possible electron hole pairs created in the InGaAs QDs do not significantly alter the device properties. However, visible light $\left(\mathrm{h} \nu>\mathrm{E}_{\mathrm{g}}\right)$ shows a strong influence over the electrical response. The last result is especially remarkable in atmospheres with high RH. Furthermore, we propose a highly efficient and sensitive humidity sensor based on InGaAs SQDs.

\section{Experimental procedure}

Two samples containing an uncapped $\operatorname{In}_{0.5} \mathrm{Ga}_{0.5} \mathrm{As}$ SQW and an $\mathrm{In}_{0.5} \mathrm{Ga}_{0.5} \mathrm{As}$ SQD layer were compared to demonstrate the high importance of the surface-to-volume ratio for the sensing efficiency. The samples were grown by means of a RIBER-32 solid-source molecular beam epitaxy (MBE) system on $n$ doped GaAs (100) substrates. In every sample, following the oxide desorption, a $1.5 \mu \mathrm{m}$ intrinsic GaAs buffer layer was grown at $590{ }^{\circ} \mathrm{C}$ and $1 \mathrm{ML} \mathrm{s}^{-1}$. Then, a layer consisting of an $\mathrm{In}_{0.5} \mathrm{Ga}_{0.5} \mathrm{As} \mathrm{SQW}$ or SQD was grown at a low substrate temperature $\left(430^{\circ} \mathrm{C}\right)$ and a a slow growth rate $\left(0.07 \mathrm{ML} \mathrm{s}^{-1}\right)$. The deposited material was $6 \mathrm{ML}$ in the case of SQD and 2.8 ML for SQW. The corresponding critical thickness for the two-dimensional (2D) to three-dimensional (3D) transition is 4.4 ML. Afterwards, substrate temperature was rapidly dropped $100{ }^{\circ} \mathrm{C}$ and then raised again to $430{ }^{\circ} \mathrm{C}$ within $3 \mathrm{~min}$. Finally, the samples were cooled down after $5 \mathrm{~s}$. The QD layer was formed by the Stranski-Krastanov method, and the growth was monitored by in situ reflection high-energy electron diffraction (RHEED).

Structural and electrical characterization was performed by means of standard atomic force microscopy (AFM) in tapping mode and a semiconductor parameter analyzer (HP4145), respectively. In order to assess the analysis of the electrical response under different energy illumination and relative humidity conditions, we designed a specific setup. It consists of an opaque and hermetically closed box where the sample is placed. This box is provided with an inlet for the gas mixture, a hygrometer to monitor the RH inside the cavity, and a light emitting diode (LED) illuminating the sample. The RH into this box is controlled by introducing a mixture of dry $\mathrm{N}_{2}$ and $\mathrm{N}_{2}$ saturated with water vapor. The percentage of moisture is controlled by two needle valves that perfectly allow a fine setting of the water vapor concentration in the final gas. Concerning the $\mathrm{I}-\mathrm{V}$ measurements, we used two metal $150 \mu \mathrm{m}$-side square contacts of $\mathrm{Ti} / \mathrm{Au}$ separated $70 \mu \mathrm{m}$ [see schema in figure 1(a)]. The contacts were not annealed to ensure conduction through the wetting and SQDs layers. Samples were encapsulated in a TO-8 to facilitate the measurement. Different illumination energy was procured by means of an infrared LED and two visible light (red and blue) LEDs for low and high irradiation energy, respectively. A Keithely 220 programmable current source was used to induce different LED powers, allowing the analysis of the electrical response as a function of the power of the incident light.

\section{Results and discussion}

Figure 1(b) shows the surface morphology of the SQD sample. As can be appreciated in the 3D AFM image, the sample contains QDs with high degree of size homogeneity and surface density $\left(7.1 \times 10^{10} \mathrm{~cm}^{-2}\right)$ and, on average, their height and base length are $6.2 \pm 0.1 \mathrm{~nm}$ and $33 \pm 4 \mathrm{~nm}$, respectively. Conversely, the SQW sample is found to exhibit a nearly flat surface with an average roughness of $0.3 \mathrm{~nm}$.

An electrical analysis as a function of the incident light power and relative humidity conditions was performed in both the InGaAs SQW and SQDs samples. It is noteworthy that temperature could significantly affect the electrical response of the sample; thus, all the experiments were conducted at a temperature of $24^{\circ} \mathrm{C}$. Device-heating effects during illumination are discarded due to the low powers used. I-V characteristics were measured under a constant incident blue-light power and by varying the relative humidity into the cavity from dry $(0 \% \mathrm{RH})$ to $60 \%$ (saturated region: it was previously reported that above $50 \%$ of RH, but SR does not exhibit any further reduction [13]). Such a sequence was repeated for several LED powers: at 0 (darkness), 1.6, 4.0, 7.4, 11.0 and $12.4 \mu \mathrm{W}$. Therefore, two agents, RH and the incidence of light, can be inferred as being responsible for the change of the SR (SR) in this experiment. Figures 2(a) and (b) show a comparison of the corresponding SR of SQW and SQDs, respectively, for every set of conditions for a constant bias voltage of $-2 \mathrm{~V}$. Consistent with this comparison, a high importance of the surface morphology can be inferred. The SQW sample does not exhibit any influence from the two mentioned parameters; there is no link between the SR and the local moisture, and the photo-to-dark SR variation is very small and remains mostly constant $(\sim 0.3 \mathrm{M} \Omega)$, with the RH as revealed in figure 2(a). On the contrary, the SQD sample shows a strong correlation between the variation of the SR and both RH and incidence of light [see figure 2(b)]. Under dry conditions and in spite of the power of the incident light, the surface conductivity persists nearly unalterable when changing from dark to photo conditions. However, when increasing the humidity in the environment, it is observed a gradual SR decay of one order of magnitude from dark to light conditions. In a high-moisture environment (i.e. 60\%), such a ratio corresponds to $\sim 49$. Two different tendencies of SR tightly related to the external agents can be also recognized in figure 2(b). First trend is linked with the illumination. For certain humidity and under lighting conditions, SR slightly varies with the power of the impinging light. The second tendency is connected to the local moisture in the environment. At a constant incident power, the SR gradually 


\section{(a)}

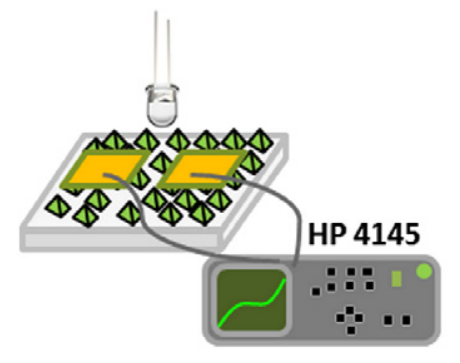

(b)

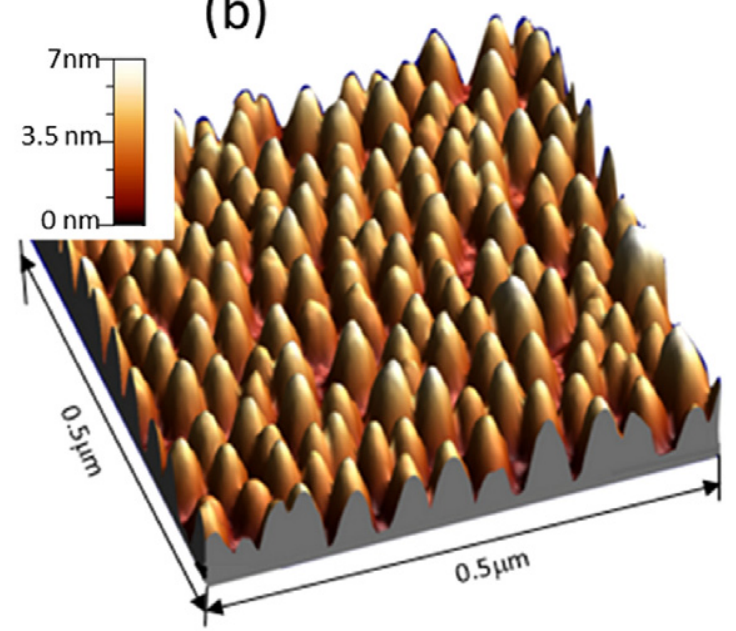

Figure 1. (a) Schematic of the processing for I-V measurements (two square Schottky junctions with $70 \times 70 \mu \mathrm{m})$. (b) $\left(0.5 \times 0.5 \mu \mathrm{m}^{2}\right) 3 \mathrm{D}$ AFM image of SQDs. The QD density is $7.1 \times 10^{10} \mathrm{~cm}^{-2}$ and the average size is $33 \pm 4 \mathrm{~nm}$ diameter and $6.2 \pm 0.1 \mathrm{~nm}$ height.
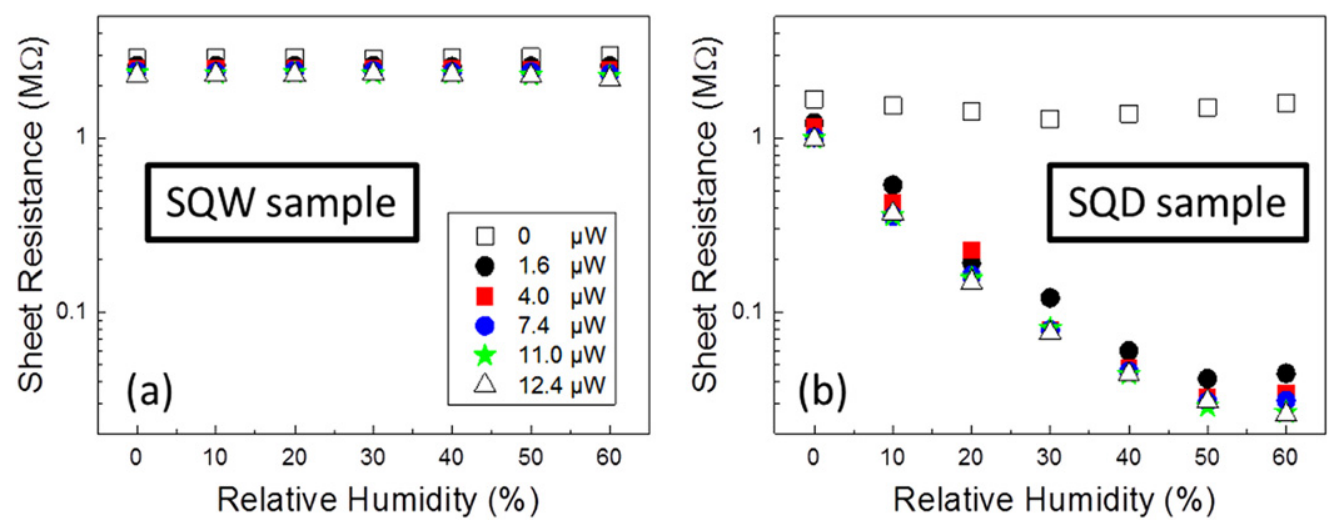

Figure 2. SR variation as a function of the red LED power and RH in the atmosphere at a bias voltage of $-2 \mathrm{~V}$. Response of a $2 \mathrm{D}$ nanostructure (a) and a SQDs sample (b). SR suffers a negligible variation in case of SQW, whereas the SR change is significant in a 3D nanostructure.

decreases with the RH towards its saturation value at $50 \%$ $\mathrm{RH}$, remaining almost unchangeable for higher relative moistures.

According to these results, it can be concluded that the change of SR requires both a humid atmosphere and photoexcitation. The adhesion of water molecules onto the surface would contribute to surface passivation, decreasing the density of active surface states, and thus allowing the motion of photoexcited carriers through the surface, resulting in an improvement of the surface conductivity. $\mathrm{RH}$ in the atmosphere is directly related to the number of water molecules in the surroundings, and thus with the probability of water adsorption onto the surface. Therefore, assuming surface states as one of the main responsible for surface behavior, it can be inferred that SQW inactivity could be tightly linked with the lower density of surface states of 2D nanostructure compared to that of the 3D. Moreover, photoexcited carriers could lead to changes in the surface energy band bending $[20,21]$, as will be discussed later. Consequently, the reduction of the density of surface states due to water adsorption in addition to the photocarriers arisen from irradiation could improve the sensitivity of the sample.

To analyze the photoexcitation process, we performed an electrical characterization of a SQDs sample using light with different energies by means of an infrared $\left(\mathrm{h} \nu<\mathrm{E}_{\mathrm{g}, \mathrm{GaAs}}\right)$, a blue and a red $\left(\mathrm{h} \nu>\mathrm{E}_{\mathrm{g}}\right)$ LED. SR as a function of the applied bias was previously found to present a symmetrical response [13]. Thus, as a representative value, in this study we focus on the reverse bias region in order to perform a narrower analysis. Figure 3(a) shows the SR curves of the SQD sample as a function of the reverse applied bias under infrared irradiation (green open stars), red light (red open triangles) and blue light (blue solid triangles) at a saturated $\mathrm{RH}$ of $50 \%$. For reference, the SR in darkness at the same RH of 50\% (open squares) is also shown. It can be observed there is a significant difference in the electrical response with regard to the energy of the excitation light. On one hand, no shift is found from darkness to infrared irradiation $(5 \mathrm{~mA})$ conditions. On 

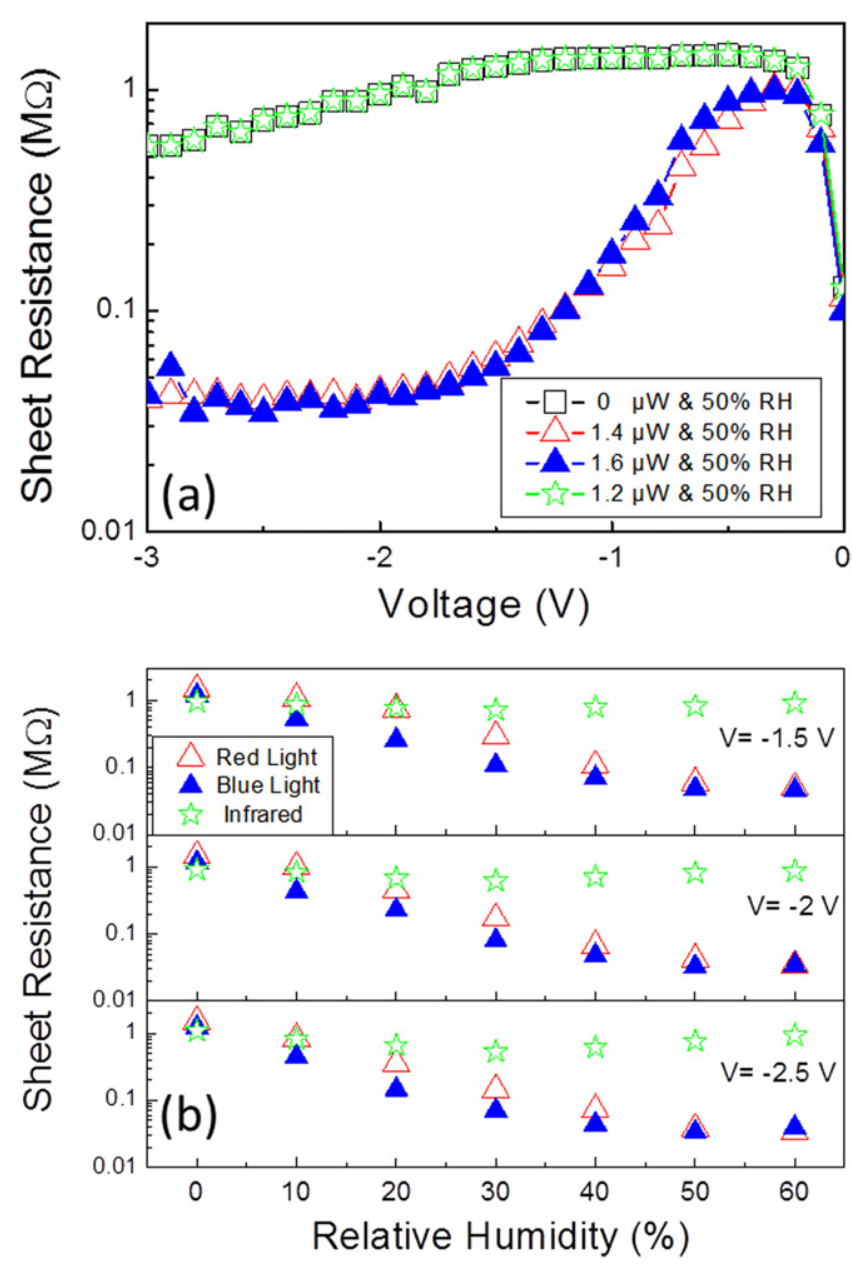

Figure 3. Electrical response of an SQD sample in different lighting and external conditions. (a) Electrical response to infrared, red and blue light (green open stars, red open and blue solid triangles, respectively) in a humid atmosphere (50\%) compared to darkness (open black squares). SR varies up to one order of magnitude when illuminating either with red or blue light. However, incidence of infrared light does not produce any change in the SR figure 3(b) shows the SR variation as a function of the applied bias at a $\mathrm{RH}$ of $50 \%$ and light powers of 1.2, 1.4 and $1.6 \mu \mathrm{W}$ for infrared, red and blue irradiation, respectively.

the other hand, under visible light, it is appreciated that there is a similar tendency of the SR with the bias voltage when illuminating either with red or blue light. For voltages above $-0.25 \mathrm{~V}$ and despite the energy of the visible light, the effect of dark-to-photo conditions at a power of $4.0 \mu \mathrm{W}$ causes an SR reduction of more than one order of magnitude. Figure 3(b) shows the SR for different values of RH, at -1.5 , -2 and $-0.25 \mathrm{~V}$ bias voltage for an infrared LED power of $1.2 \mu \mathrm{W}$ and red and blue LED powers of 1.4 and $1.6 \mu \mathrm{W}$, respectively. As can be observed, under infrared irradiation no impact of the local humidity on the electrical response is found. Conversely, the effect of the visible light is strongly dependent on the local moisture, despite its energy. In a dry atmosphere, the irradiation does not affect the SR. However, under visible light and in a humid atmosphere, the SR greatly decreases. Such a reduction is significantly noticeable at high $\mathrm{RH}$ conditions (a factor of $\sim 20$ between $\mathrm{RH}=0 \%$ and $\mathrm{RH}=$
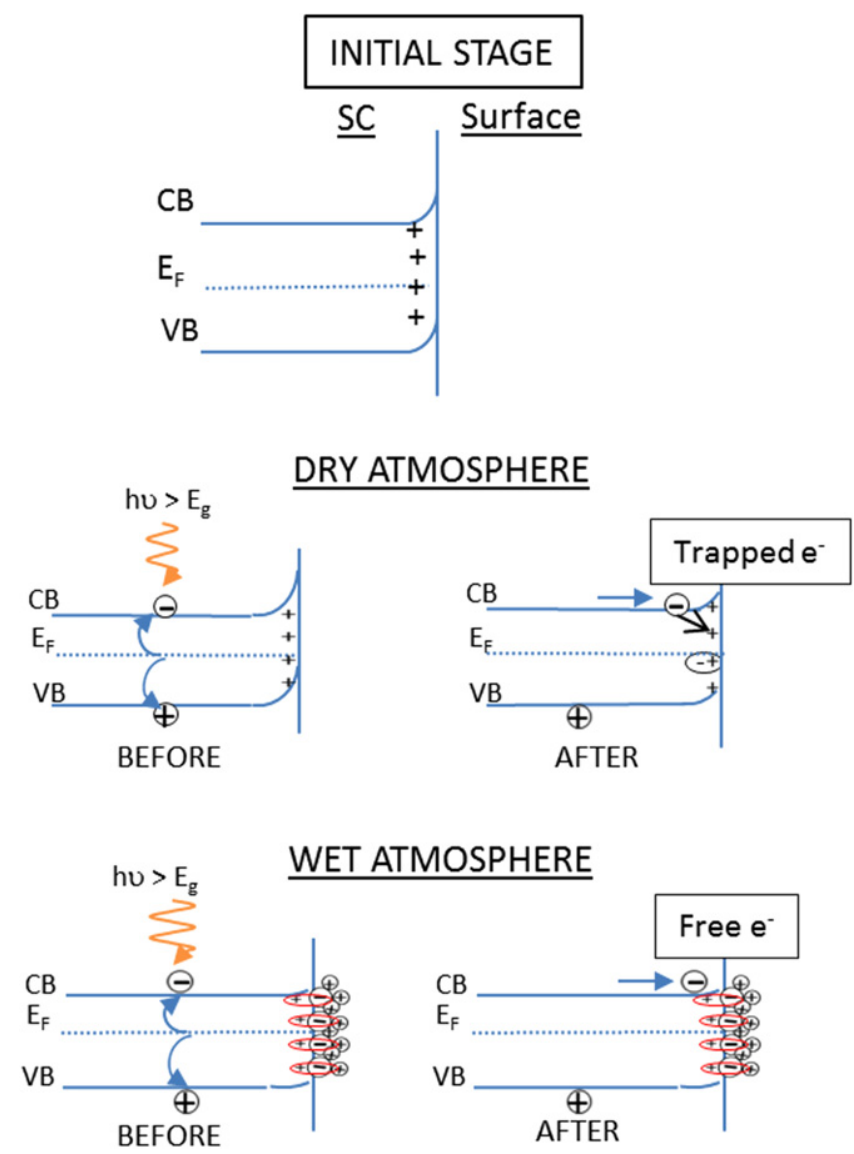

Figure 4. Schematic of the energy band bending diagrams. From an initial stage with an upward bent band, illuminating with energy larger than the bandgap evolves to lower the band bending and, under favorable external conditions (high $\mathrm{RH}$ atmospheres), to improve the surface conductivity. In a dry atmosphere, surface states are unoccupied, and then the released carrier is trapped at these centers, and hence, surface conductivity is not improved. Conversely, in a wet atmosphere, the surface states are passivated by water molecules, and thus carriers generated after illumination contribute to surface conductivity.

$60 \%$ ). These results indicate that the change in the electrical response is a consequence of a photoinduced process and it is extremely vulnerable to the molecule water percentage in the surroundings. Furthermore, the photoexcitation process that has arisen due to surface irradiation is shown to be dependent on the energy of the incoming light.

Incidence of light in a semiconductor is known to affect the electronic properties of the surface [22]. It has been demonstrated that illuminating the sample with photons with energy larger than the semiconductor bandgap strongly influences the optical and electrical properties of nanostructures with high surface to volume ratios [21, 23], likely as a consequence of a band-bending variation [24]. In our case, and assuming a positively charged surface mainly due to dangling bonds from III-group elements [12], it is plausible to accept an initial stage of upward band bending as depicted in figure 4. According to the experiments, the energy of the impinging light is found to play a meaningful role in its impact on the electrical characteristics. 
Illuminating with excitation energy below the $\mathrm{E}_{\mathrm{g}, \mathrm{GaAs}}$ affects a small absorption volume and thus, a low density of carriers could be activated, leading to an insignificant contribution to the conductivity. Nonetheless, irradiating with an energy larger than the $\mathrm{E}_{\mathrm{g}, \mathrm{GaAs}}$ can excite a high density of photocarriers that could reach the surface as free carriers and therefore improve the surface conductivity under favorable moist conditions. Figure 4 shows a diagram of the energy bands under dry and wet conditions (top and bottom figures, respectively). In a dry atmosphere, the SR remains mostly constant regardless of the lighting. The surface states are unoccupied, and therefore, they are active trapping and recombination centers. When the semiconductor is illuminated, a density of electron-hole pairs is generated in the bulk. Although electrons could be attracted by the positively charged surface states, they hardly move towards the surface because of the presence of a high potential energy barrier at the interface and thus, only a few electrons can reach the surface. Moreover, those arriving to the surface could be likely trapped by the active centers, and therefore will not contribute to increase the conductivity. Conversely, in a wet atmosphere the surface states are presumed to be linked to adsorbed water molecules. It has been previously addressed that water physisorption yields to an improvement of the electrical and optical characteristics, decreasing the SR and increasing the photoluminescence, respectively [10-13]. This has been attributed to a reduction of the density of active surface states after this physical process, in which the negative part of the water molecule dipole is attached to the positively charged surface states. Water would therefore passivate the empty surface states and consequently reduce the surface band bending (see the lower part of figure 4). As a result, the photoexcited electrons could now reach more easily the surface. In addition, since surface states are passivated, carriers would unlikely be trapped, and can therefore contribute to increase the surface conductivity.

\section{Conclusions}

In conclusion, we conducted a detailed analysis of the impact of the illumination on the electrical response of $\operatorname{In}_{0.5} \mathrm{Ga}_{0.5} \mathrm{As}$ surface nanostructures (quantum wells and quantum dots) with different relative humidity in the atmosphere. The results once more highlight the importance of the surface-to-volume ratio for sensing applications. $2 \mathrm{D}$ nanostructures suffer a negligible change in the surface conductivity with both agents: illumination and relative humidity. Nevertheless, an SR decay of one order of magnitude at high relative humidity from dark-to-photo conditions in SQD was found. Furthermore, it was shown that the incidence of light plays a significant role in the electrical properties. Irradiation with energy below the semiconductor bandgap causes an insignificant effect on surface conductivity, whereas an incoming light with energy larger than the bandgap yields to a relevant SR variation. Such a SR alteration is also demonstrated to be vulnerable to local humidity conditions, remaining nearly constant in dry atmospheres and reducing up to one order of magnitude in moist environments. We suggest that the incidence of light generates electron-hole pairs that could contribute to the surface conductivity only under favourable external conditions. The adhesion of water molecules onto the surface reduces the density of active surface states and the surface band bending, facilitating electron motion and therefore improving the electrical surface sensitivity. These results make $\mathrm{In}_{0.5} \mathrm{Ga}_{0.5} \mathrm{As}$ SQD nanostructures very promising for the development of humidity sensor devices.

\section{Acknowledgments}

This work has been supported by the Comunidad de Madrid through project P2009/ESP-1503.

\section{References}

[1] Chen M, Kobashi K, Chen B, Lu M and Tour J M 2010 Functionalized self-assembled InAs/GaAs quantum-dot structures hybridized with organic molecules $A d v$. Funct. Mater. 20 469-75

[2] Jun B-H et al 2012 Ultrasensitive, biocompatible, quantumdot-embedded silica nanoparticles for bioimaging $A d v$. Funct. Mater. 22 1843-9

[3] Sailor M J and Lee E J 1997 Surface chemistry of luminescent silicon nanocrystallites Adv. Mater. 9 783-93

[4] Xue X, Nie Y, He B, Xing L, Zhang Y and Wang Z L 2013 Surface free-carrier screening effect on the output of a $\mathrm{ZnO}$ nanowire nanogenerator and its potential as a self-powered active gas sensor Nanotechnology $\mathbf{2 4} 225501$

[5] Guo L et al 2012 Two-beam-laser interference mediated reduction, patterning and nanostructuring of graphene oxide for the production of a flexible humidity sensing device Carbon NY 50 1667-73

[6] Zhang X, Ming H, Liu R, Han X, Kang Z, Liu Y and Zhang Y 2013 Highly sensitive humidity sensing properties of carbon quantum dots films Mater. Res. Bull. 48 790-4

[7] Liang B L, Wang Z M, Mazur Y I and Salamo G J 2006 Photoluminescence of surface InAs quantum dot stacking on multilayer buried quantum dots Appl. Phys. Lett. 89243124

[8] Liang B L, Mazur Y I, Kunets V P, Wang Z M, Salamo G J, DeCuir E a Jr, Passmore B and Manasreh M O 2008 Enhanced photoluminescence from InAs/GaAs surface quantum dots by using a Si-doped interlayer Nanotechnology 19065705

[9] Moison J, Elcess K, Houzay F, Marzin J, Gérard J, Barthe F and Bensoussan M 1990 Near-surface GaAs/ Ga0.7Al0.3As quantum wells: interaction with the surface states Phys. Rev. B 41 12945-8

[10] Saito H, Nishi K and Sugou S 1998 Influence of GaAs capping on the optical properties of InGaAs/GaAs surface quantum dots with $1.5 \mu \mathrm{m}$ emission Appl. Phys. Lett. 732742

[11] Milla M J, Ulloa J M and Guzmán Á 2012 High optical sensitivity to ambient conditions of uncapped InGaAs surface quantum dots Appl. Phys. Lett. 100131601

[12] Milla M J, Ulloa J M and Guzmán Á 2013 Dependence of surface ingaas quantum dot luminescence on the molecular properties of the environment Appl. Phys. Expr. 6092002

[13] Milla M J, Ulloa J M and Guzmán Á 2014 Strong influence of the humidity on the electrical properties of InGaAs surface quantum dots. ACS Appl. Mater. Interfaces 6 6191-5 
[14] Duijs E F, Findeis F, Deutschmann R A, Bichler M, Zrenner A, Abstreiter G, Adlkofer K, Tanaka M and Sackmann E 2001 Influence of thiol coupling on photoluminescence of near surface InAs quantum dots Phys. Status Solidi. 224 871-5

[15] Miao Z, Chen P, Lu W, Xu W, Li Z, Cai W, Shi G and Shen S 2000 Study on the confined states in single surface quantum wells Phys. Lett. A 273 271-5

[16] Wang J Z, Yang Z, Yang C L and Wang Z G 2000 Photoluminescence of InAs quantum dots grown on GaAs surface Appl. Phys. Lett. 772837

[17] Krzyzewski T J and Jones T S 2004 Ripening and annealing effects in InAs/GaAs(001) quantum dot formation $J$. Appl. Phys. 96668

[18] Kang B S, Louche G, Duran R S, Gnanou Y, Pearton S J and Ren F 2004 Gateless AlGaN/GaN HEMT response to block co-polymers Solid. State. Electron. 48 851-4

[19] Neuberger R, Müller G, Ambacher O and Stutzmann M 2001 High-electron-mobility AlGaN/GaN transistors (HEMTs) for fluid monitoring applications Phys. Status Solidi 185 85-9

[20] Watson D F and Meyer G J 2005 Electron injection at dyesensitized semiconductor electrodes Annu. Rev. Phys. Chem. 56 119-56

[21] Zhang Z and Yates J T 2012 Band bending in semiconductors: chemical and physical consequences at surfaces and interfaces Chem. Rev. 112 5520-51

[22] Yin H, Li T, Hu W, Wang W, Li N, Chen X and Lu W 2010 Nonequilibrium carrier distribution in semiconductor photodetectors: surface leakage channel under illumination Appl. Phys. Lett. 96263508

[23] Bera A and Basak D 2008 Carrier relaxation through twoelectron process during photoconduction in highly UV sensitive quasi-one-dimensional $\mathrm{ZnO}$ nanowires Appl. Phys. Lett. 93053102

[24] Ashkenasy G, Cahen D, Cohen R, Shanzer A and Vilan A 2002 Molecular engineering of semiconductor surfaces and devices Acc. Chem. Res. 35 121-8 